\title{
Photoluminescence and Intrinsic Properties of MBE-Grown InN Nanowires
}

\author{
Toma Stoica, ${ }^{, \dagger}$ Ralph J. Meijers, ${ }^{\dagger}$ Raffaella Calarco, ${ }^{\dagger}$ Thomas Richter,${ }^{\dagger}$ \\ Eli Sutter, ${ }^{\ddagger}$ and Hans Lüth ${ }^{\dagger}$
}

Institute of Thin Films and Interfaces (ISG1) and Center of Nanoelectronic Systems for Information Technology (cni), Research Centre Jülich, 52425 Jülich, Germany, and Center for Functional Nanomaterials, Brookhaven National Laboratory, Upton, New York 11973

Received March 10, 2006; Revised Manuscript Received June 6, 2006

\begin{abstract}
The influence of the growth parameters on the photoluminescence (PL) spectra has been investigated for samples with columnar morphology, either with InN columns on original substrates or as free-standing nanowires. Valuable information about band gap and electron concentration was obtained by line shape analysis. Optical band gaps between 730 and $750 \mathrm{meV}$ and electron concentrations of $8 \times 10^{17}$ to $6 \times 10^{18} \mathrm{~cm}^{-3}$ were derived from the fit of the PL spectra of different samples. The crystalline quality of the wires was investigated by high-resolution transmission electron microscopy.
\end{abstract}

Nanowires are intensively studied for future device applications of low-dimensional systems. ${ }^{1}$ Within the nitride group, $\mathrm{InN}$ is an interesting material because of its high electron mobility, its surface accumulation layer, ${ }^{2}$ low band gap, and low toxicity. ${ }^{3}$ InN nanowires were prepared from indium metal and ammonia using a vapor-solid growth mechanism with or without $\mathrm{Au}$-nanoparticle catalysis ${ }^{4-6}$ or with the help of a porous alumina template. ${ }^{7,8}$

Plasma-assisted molecular beam epitaxy (PA-MBE) was also successfully applied to produce $\mathrm{InN}$ nanocolumns. ${ }^{9-11}$ As for other nitride semiconductors, the growth parameters are adjusted to nitrogen-rich conditions to obtain columnar structures, with well-separated and uniform-in-diameter columns and with a relatively good crystalline quality. ${ }^{11}$ Freestanding nanowires (FSNWs) can easily be obtained by removal of the columns from their original substrate.

Despite the assiduous scientific effort, one of the basic properties of InN, the band gap, is still under debate. It seems that the optical properties of $\mathrm{InN}$ are very much dependent on the deposition method and the intrinsic doping concentration. An improvement of the film quality has been obtained using PA-MBE ${ }^{12}$ and subsequently a smaller gap of about 1 $\mathrm{eV}$ or at least less than $1 \mathrm{eV}$ has been reported. ${ }^{13,14}$ This is in disagreement with the earlier reported value of about 2 $\mathrm{eV} .{ }^{15}$ Efforts are made ${ }^{3,16-20}$ to understand the difference between InN with an optical band gap lower than $1 \mathrm{eV}$,

* To whom correspondence should be addressed. E-mail: t.stoica@ fz-juelich.de.

Institute of Thin Films and Interfaces (ISG1) and Center of Nanoelectronic Systems for Information Technology (cni), Research Centre Jülich.

$\doteqdot$ Center for Functional Nanomaterials, Brookhaven National Laboratory. recently obtained by PA-MBE, ${ }^{9,10,13,14,21-26}$ but also with metal organic chemical vapor deposition (MOCVD), ${ }^{6,27}$ and InN layers with an apparently higher band gap of about 2 $\mathrm{eV}$ obtained with different deposition methods and reported within earlier papers, as well as in more recent publications. . $^{4,5,7,8,19,28,29}$

Within this paper we have investigated photoluminescence (PL) properties of InN layers obtained by PA-MBE on $\mathrm{Si}(111)$ substrates. The studies are restricted to samples with columnar growth, applicable for fabrication of FSNWs by the removal of columns from their original substrates. For the investigation of optical properties, PL is an efficient tool, especially for samples with relatively well-separated columns for which normal transmission measurements are difficult. The aim of this paper is a detailed analysis of the PL spectra obtained under different measurement conditions to evaluate intrinsic properties of $\mathrm{InN}$ nanowires (band gap, electron concentration), for different growth parameters. Studies of the column morphology and corresponding PL properties for growth optimization are published in ref 11.

Radio frequency PA-MBE was used for columnar growth of $\mathrm{InN}$ on $\mathrm{Si}(111)$, at temperatures within the range $440-$ $525{ }^{\circ} \mathrm{C}$. A $\mathrm{N}_{2}$ flux of $4.0 \mathrm{sccm}$ and a plasma cell forward power of $500 \mathrm{~W}$ were applied. The In flux was varied by changing the beam equivalent pressure (BEP) within the range $(2.3-15.0) \times 10^{-8}$ mbar. Growth parameters of the investigated samples are shown in Table 1. More details about the growth conditions can be found in ref 11. In Table 1 , results of the intrinsic properties found by a modeling of the PL spectra (see below) are also included. 
Table 1. Growth Parameters and Intrinsic Properties of Different InN Nanocolumnar Samples ${ }^{a}$

\begin{tabular}{lcccccc}
\hline & $\begin{array}{c}T_{\text {subs }} \\
\text { sample }\end{array}$ & $\begin{array}{c}\text { In-BEP } \\
\left(\times 10^{-8} \mathrm{mbar}\right)\end{array}$ & $\begin{array}{c}t_{\text {dep }} \\
(\mathrm{min})\end{array}$ & $\begin{array}{c}L \\
(\mu \mathrm{m})\end{array}$ & $\begin{array}{c}E_{\mathrm{g}} \\
(\mathrm{meV})\end{array}$ & $\begin{array}{c}n_{\mathrm{e}} \\
\left(\times 10^{18} \mathrm{~cm}^{-3}\right)\end{array}$ \\
\hline NC58 & 475 & 2.3 & 120 & 0.25 & 738 & 5.5 \\
NC56 & 475 & 2.8 & 120 & 0.75 & 734 & 6.0 \\
NC57 & 475 & 3.9 & 120 & 0.55 & 737 & 4.2 \\
NC44 & 475 & 7.0 & 120 & 0.75 & 738 & 2.4 \\
NC45 & 475 & 10.0 & 120 & 0.86 & 742 & 1.7 \\
NC46 & 475 & 15.0 & 120 & 1.10 & 738 & 1.2 \\
NC33 & 525 & 3.9 & 240 & 1.80 & 744 & 0.8 \\
NC31 & 475 & 2.3 & 240 & 0.80 & 742 & 3.8 \\
NC32 & 475 & 3.9 & 240 & 1.10 & 736 & 2.5 \\
NC59 & 440 & 3.9 & 240 & 0.54 & 754 & 3.2 \\
FSNW31 & 475 & 2.3 & 240 & 0.80 & 743 & 5.2 \\
FSNW33 & 525 & 3.9 & 240 & 1.80 & 742 & 1.4
\end{tabular}

${ }^{a}$ Deposition temperature $T_{\text {subs }}$; In-BEP; deposition time $t_{\text {dep }}$; column length $L$; band gap $E_{\mathrm{g}}$, and electron concentration $n_{\mathrm{e}}$ obtained by a line shape analysis of PL spectra.

The photoluminescence was measured using a Fourier transform spectrometer (BIORAD FTS40) equipped with a cooled $\mathrm{Ge}$ detector and an argon ion laser emitting $50 \mathrm{~mW}$ at $488 \mathrm{~nm}$ wavelength. The laser-beam power was varied between 2 orders of magnitude by changing the laser current and/or using neutral filters. The measurements were performed in the temperature range of $4-300 \mathrm{~K}$ in a He cryostat. As deposited samples (named NCxx) as well as FSNW samples (named FSNW $x x$ ) have been investigated. FSNWs were obtained by removing the wires from the original silicon substrate and inserting them in a silicon grease on sapphire substrates. It was checked that no PL signal is measured on sapphire covered with the used type of grease, but without InN nanowires.

Transmission electron microscopy (TEM) experiments were carried out in a JEOL JEM 3000F field emission transmission electron microscope on samples obtained by dispersing the InN nanowires on a thin amorphous carbon film on a TEM grid. The experiments were carried out at very low beam intensity in order to prevent any uncontrolled structural modifications.

InN nanowires were obtained by PA-MBE under $\mathrm{N}_{2}$-rich conditions ${ }^{11}$ using different growth parameters (see Table 1). In Figure 1a, PL spectra measured at different temperatures for samples with well-defined nanocolumns are shown. A SEM lateral-side view of the columns is shown in the inset of the figure. Both phenomena of increasing and decreasing of the wire diameter from bottom to the top are observed. An atomic force microscopy (AFM) image of a single FSNW with an increase of the diameter is shown in Figure 1b. The intense PL peak at $780 \mathrm{meV}$ decreases in intensity with increasing the measurement temperature. Similar sets of spectra for different excitation powers are obtained for each sample and results of spectra analysis are listed in Table 1. PL spectra measured within the visible range show no detectable emission close to $2 \mathrm{eV}$ (see also discussions in ref 11).

To illustrate the crystalline quality of the InN wires, TEM images of FSNWs of sample NC44 are given in panels c
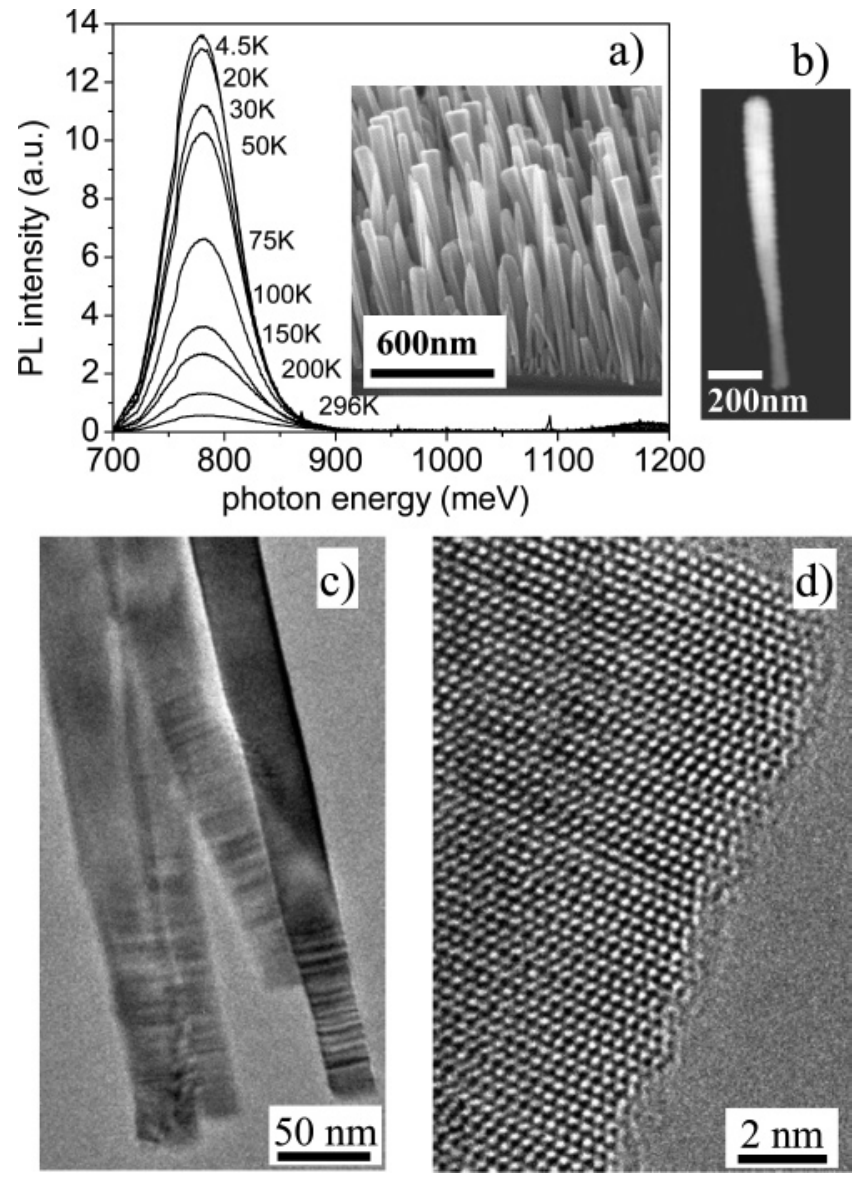

Figure 1. (a) Temperature dependence of PL spectra of sample NC44, excitation power $50 \mathrm{~mW}$. The inset shows a side view SEM image. (b) AFM image of a single FSNW. (c and d) Low- and high-magnification TEM images of nanowires of sample NC44.

and d of Figure 1. Bottom parts of a few wires are shown in Figure 1c (see also Figure 1b and the inset of Figure 1a), while the high-resolution TEM image in Figure 1d shows the crystalline structure of the wire. The bottom and top parts of the nanowires have been recognized with the help of a broadening present on the sample top. In general the nanowires are nicely crystalline but have some point defects and stacking faults more frequently at the base of the wires than at the tip. A general conclusion of TEM investigations is that the wires grow with $c$-axis parallel to the wire. More detailed analysis of the TEM investigation will be published in a forthcoming publication.

The PL efficiency increases with column length and deposition temperature and reaches a maximum for an In flux of $10^{-7}$ mbar. ${ }^{11}$ Within this paper, the dependences of PL spectra on laser excitation intensity and measurement temperature were analyzed for as-grown columnar and FSNW samples obtained under different growth conditions to gather information about the recombination process and intrinsic properties of the $\mathrm{InN}$ nanowires.

A change of the laser intensity has a weak influence on the peak position and the shape of the PL spectra. In Figure 2a, spectra of a sample with high PL efficiency FSNW33 are shown for different laser powers, normalized for comparison of the low energy tails. The evolution of the spectra 

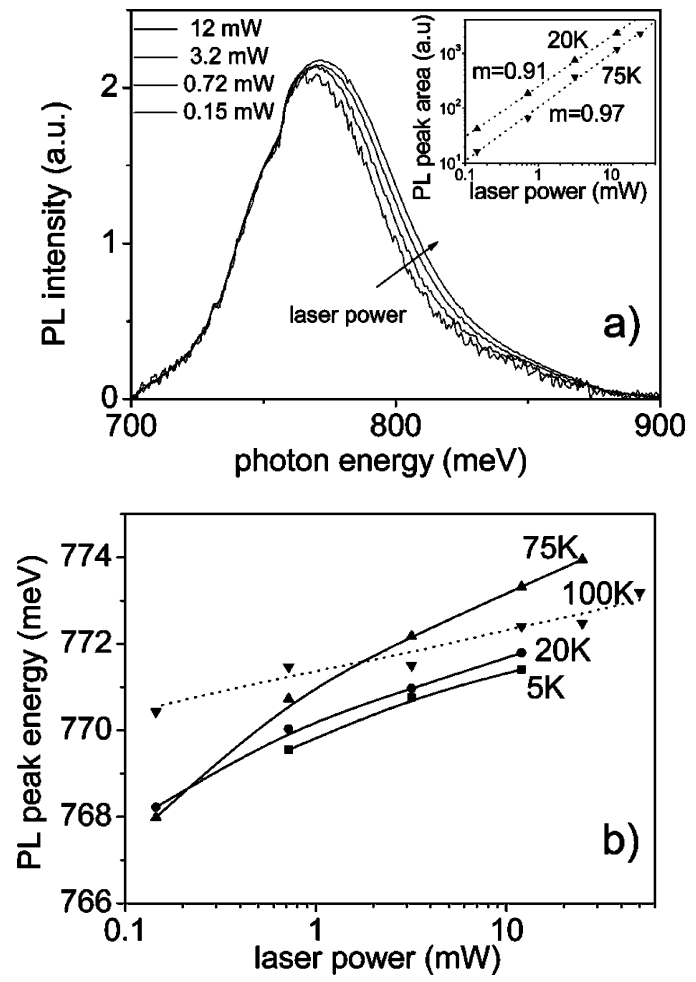

Figure 2. Laser power dependence of PL spectra of a high efficiency sample FSNW33: (a) Normalized spectra for different excitation power, at $20 \mathrm{~K}$. The inset shows the laser power dependence of the peak intensity for two measurement temperatures. (b) Laser power dependence of the peak position for different temperatures.

shows a weak band filling effect due to photocarriers, i.e., a small broadening and blue shift of the peak with excitation power. For InN as a degenerate n-type semiconductor, the band filling effect should be attributed mostly to the minority band of holes. The PL intensity varies almost linearly with the excitation power (inset, Figure 2a). The area of the PL peak depends on the excitation power $\Phi$ approximately as $\sim \Phi^{m}$, with $m$ values in the range 0.9-1.0 for different samples and temperatures.

For InN layers, generally, a high equilibrium free electron density due to unintentional doping is observed. ${ }^{23}$ Therefore, the linear dependence of the PL intensity vs excitation power is easily explained by the weak excitation conditions in our experiments, corresponding to a low photocarrier concentration relative to the background doping. The weak band filling effect shows up as a small change of the PL peak position with laser power. For the sample in Figure $2 b$, the peak energy varies less than $6 \mathrm{meV}$ for a change of the laser power by more than 2 orders of magnitude (maximum shift is obtained at $75 \mathrm{~K}$ ). One reason for the weak laser-power dependence of the shape of the PL spectra is a large broadening of the PL peak due to fluctuations along the wires and between wires, which might hide the local band filling effect. The logarithmic dependence on laser power and the small blue shift with temperature observed up to $75 \mathrm{~K}$ (Figure $2 b)$ are in agreement with a distribution of holes in an exponential tail of localized states (Urbach tail) at the edge of the valence band. ${ }^{26}$ At low temperatures, the holes are
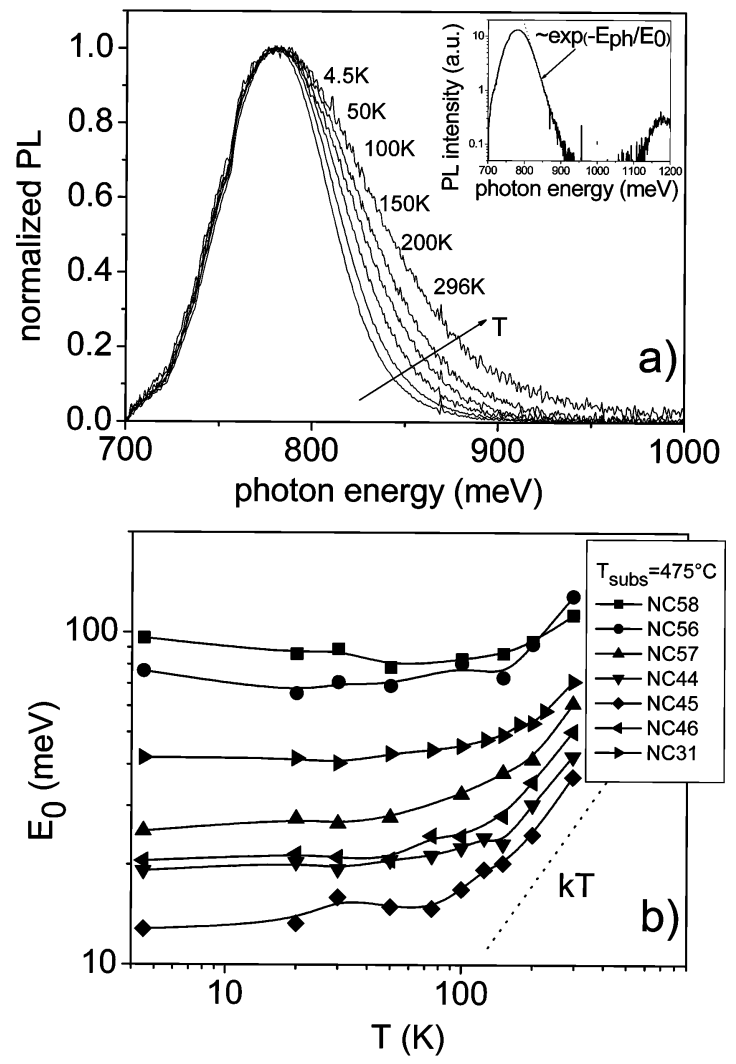

Figure 3. (a) Normalized PL spectra at different measurement temperatures, for sample NC44 using a laser power of $50 \mathrm{~mW}$. The inset with the spectrum at $20 \mathrm{~K}$ on a logarithmic scale shows the exponential dependence of the tail at the high photon energy side of the main peak, with the specific energy $E_{0}$. (b) Temperature dependence of the specific energy $E_{0}$ for different samples (only the data for samples deposited at $475^{\circ} \mathrm{C}$ are shown). The linear function $k T$ is included for comparison.

excited from localized states to the valence band upon increasing the temperature. As a result, the PL peak shifts to higher energy. The width of the tail of states should be of the order of a few millielectronvolts, which corresponds to the observed blue shift at low temperatures.

PL spectra normalized for comparison of the low energy tails are shown for different measurement temperatures in Figure 3a for sample NC44. As we can see, the highenergy tail is more extended for higher temperatures. This tail has an exponential dependence on photon energy $\sim \exp \left(-E_{\mathrm{ph}} / E_{0}\right)$ with a specific energy $E_{0}$ and $E_{\mathrm{ph}}$ as photon energy (inset of Figure 3a). The temperature dependence of $E_{0}$ is shown in Figure $3 \mathrm{~b}$ for different samples grown at 475 ${ }^{\circ} \mathrm{C}$. Because the photocarriers excited into bands are rapidly thermalized, the high-energy tail of the PL spectra reflects the thermal distribution of the carriers above the Fermi level and $E_{0}$ should correlate with the thermal energy $k T$ of the Boltzmann factor (in Figure $3 \mathrm{~b}$ the function $k T$ is shown for comparison). The $k T$ dependence is approximately found only for higher measurement temperatures. Up to $100 \mathrm{~K}$, the energy $E_{0}$ has almost constant values within the range 10$100 \mathrm{meV}$, varying from sample to sample. This $E_{0}$ value at low temperatures reflects the fluctuations in nanocolumn properties. A smaller value corresponds to a higher quality of the columns. We can see in Figure $3 \mathrm{~b}$ that smaller $E_{0}$ 
a)

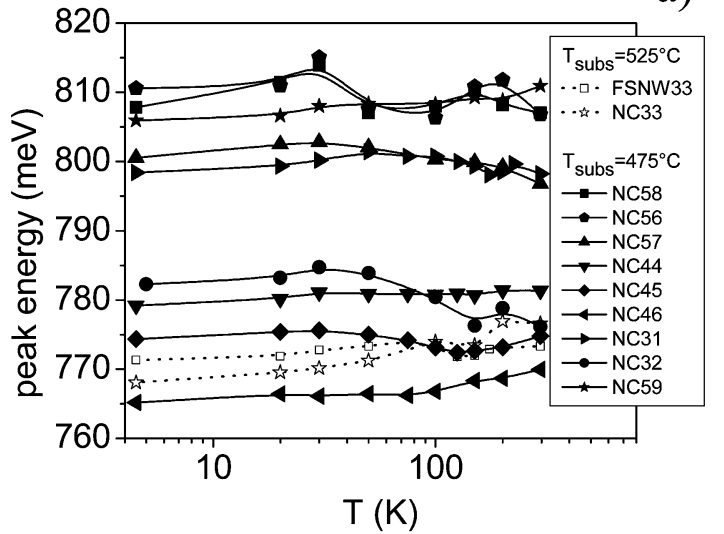

c)

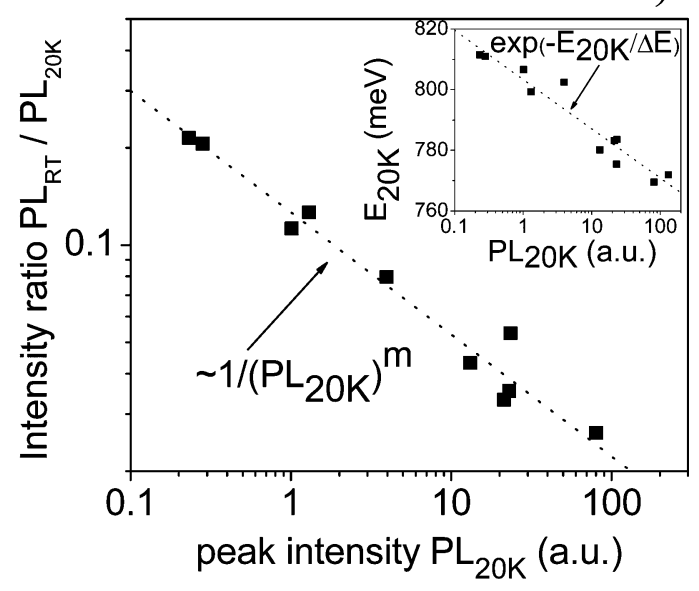

b)

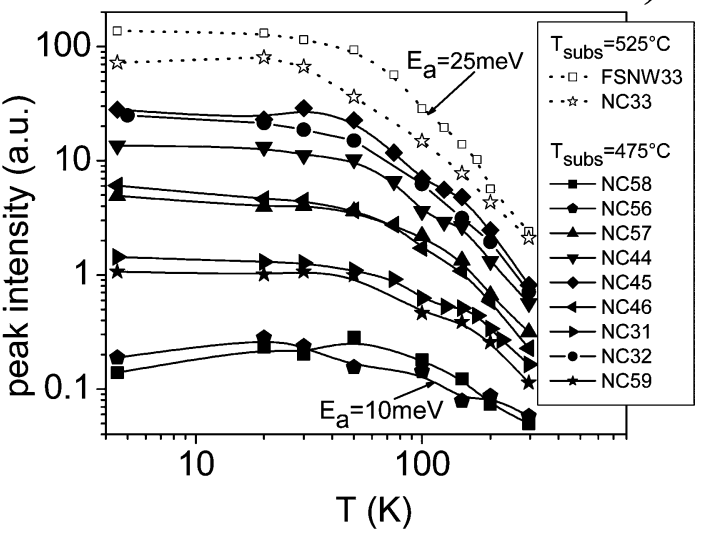

d)

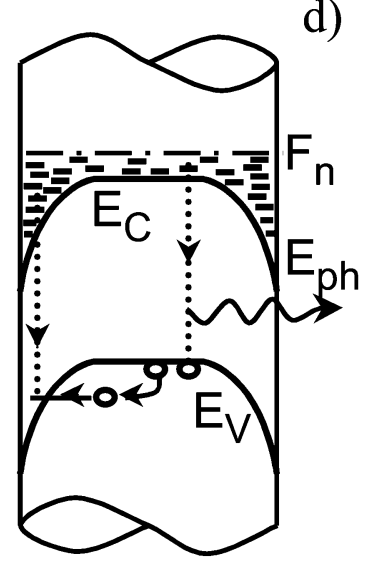

Figure 4. Temperature dependence of PL for samples deposited at 475 and $525^{\circ} \mathrm{C}$ : (a) peak energy; (b) peak intensity; (c) ratio of peak intensity at RT and $20 \mathrm{~K}$ vs intensity at $20 \mathrm{~K}$. The inset shows the correlation of peak energy and peak intensity at $20 \mathrm{~K}$. The excitation power was $50 \mathrm{~mW}$. In part $\mathrm{d}$ a schematic band diagram illustrates the carrier recombination by thermal activation and tunneling into the accumulation layer of the wire surface $\left(E_{\mathrm{C}}\right.$ and $E_{\mathrm{V}}$ are conduction and valence edges, $F_{\mathrm{n}}$ is the Fermi level in the conduction band).

values are obtained for samples NC45 and NC44. These samples exhibit the optimal wire morphology at a growth temperature of $475{ }^{\circ} \mathrm{C} .{ }^{11}$ Beside fluctuations, Landsberg broadening ${ }^{33}$ may add a component to $E_{0}$, which is more important for higher doping as discussed below.

The temperature dependence of the peak energy yields information about the temperature dependence of the band gap. But, there are also other effects, which might have an influence on this dependence, as for example the carrier redistribution on electronic states and/or to different sites of a nonuniform sample. Therefore, for relatively large fluctuations within a sample with many $\mathrm{InN}$ nanocolumns, the peak energy vs temperature dependence shows a different behavior: the peak energy is almost constant, increasing or decreasing with temperature, as can be seen in Figure 4a.

A superposition of the PL emission of higher and lower doping regions can essentially influence the temperature dependence of the peak position. We can see from parts $b$ and $\mathrm{c}$ of Figure 4 that a higher emission (lower electron concentration, lower peak energy) corresponds to a more rapid decreasing of the intensity with the temperature than for lower emission cases (higher electron concentration, higher energy peak). So, at higher temperatures the contribution of the emission with a higher energy peak becomes more important and may induce an apparent blue shift which can compensate a normal red shift due to the thermal dependence of the band gap. We discuss in the following the origin of this correlation of PL intensity and its thermal dependence. An unusual temperature dependence of the PL peak energy was also reported for $\mathrm{InN}$ in ref 30.

For most samples, there is an initial increase of the peak energy at low temperatures up to $75 \mathrm{~K}$, followed by a slow decrease at higher temperatures as can be seen in Figures $2 \mathrm{~b}$ and $4 \mathrm{a}$. The influence of a tail of states of the valence band can explain this increase of the peak energy at low temperatures.

The temperature dependence of the peak intensity is shown in Figure $4 \mathrm{~b}$ for different samples. The intensity decreases with an activation energy $E_{\mathrm{a}}$ between 10 and $25 \mathrm{meV}$. Higher $E_{\mathrm{a}}$ values are obtained for samples with higher PL efficiency. This can be described by the ratio of the PL intensity at room temperature $\left(\mathrm{PL}_{\mathrm{RT}}\right)$ and its value at $20 \mathrm{~K}\left(\mathrm{PL}_{20 \mathrm{~K}}\right)$ as a function of $\mathrm{PL}_{20 \mathrm{~K}}$. This ratio decreases as $\sim\left(\mathrm{PL}_{20 \mathrm{~K}}\right)^{-m}$ with $m \sim 0.9$ (Figure $4 \mathrm{c}$ ). The smallest value of 0.02 is obtained for sample NC33 with higher PL efficiency. The thermal quenching of the PL signal is due to the activation of photocarriers to sites or electronic states where nonradiative recombination is increased.

The observed correlation of thermal quenching with PL efficiency can be understood by means of a model based on 
accumulation layers at the column surface. Accumulation layers due to pinning of the Fermi level above the conduction band edge $e^{2,31}$ are usually found on surfaces of narrow band gap semiconductors such as InAs and InSb. Photoholes are thermally activated over the potential barrier of the downward bend valance band at the surface and recombine mostly nonradiatively with the high-density electrons of this surface accumulation layer. Due to different background doping, there are different potential barriers for holes in different samples and, as a consequence, different activation energies. A schematic band diagram to illustrate the recombination of holes by thermal activation and tunneling into the surface accumulation layer is shown in Figure 4d. The thickness of this accumulation layer is estimated to be of the order of a few nanometers. We can expect that this surface recombination process increases the nonradiative recombination due to Auger recombination and also contributes to the low energy tail of PL spectra. In the opposite case of a surface depletion layer, the tunneling effect is negligible and the total recombination within wires is reduced due to the surface barrier, as shown for $\mathrm{GaN}$ nanowires in ref 32 .

An interesting correlation was found between the peak energetic position $E_{20 \mathrm{~K}}$ and the $\mathrm{PL}_{20 \mathrm{~K}}$ intensity (inset of Figure $4 \mathrm{c}$, measurement temperature $20 \mathrm{~K}$ ). An exponential dependence $\mathrm{PL}_{20 \mathrm{~K}} \sim \exp \left(-E_{20 \mathrm{~K}} / \Delta E\right)$ with $\Delta E=7.1 \mathrm{meV}$ is a good fit to the experimental data. This correlation can give information about recombination processes. The peak energy increases with electron concentration for n-type degenerate semiconductors, because of the Fermi level shift into the conduction band. If we assume that the change from sample to sample is due to variations of the electron concentration (n-doping) and that the concentration of nonradiative centers is constant, then the PL intensity is nearly constant for a Schottky-Read-Hall recombination and decreases with the electron density, in the case of a dominant Auger recombination (see below).

The movement of the Fermi level within the conduction band for a degenerate semiconductor like $\mathrm{InN}$ results in changes of the PL peak position. A first-approximation model for describing the shape of PL spectra is based on a formula for recombination of photogenerated holes and electrons in the Fermi sea of the conduction band. However, such a simple model does not fit well with the experimental spectra. At low temperatures, the PL spectra show a longer tail at the high energy side than expected solely from the thermal distribution of carriers (see Figure $3 \mathrm{c}$ and related discussions). Therefore, additional broadening effects should be included in the model to evaluate the Fermi level position.

The spectra can be modeled using the convolution of the carrier distribution in the conduction $\left(\rho_{\mathrm{C}}\right)$ and valence $\left(\rho_{\mathrm{V}}\right)$ bands with a broadening function $P$

$$
\begin{aligned}
I_{\mathrm{ph}}\left(E_{\mathrm{ph}}\right) \sim & \int \rho_{\mathrm{C}}\left(\epsilon_{\mathrm{n}}, \epsilon_{\mathrm{Fn}}\right) \rho_{\mathrm{V}}\left(\epsilon_{\mathrm{p}}, \epsilon_{\mathrm{Fp}}\right) \\
& P\left(E_{\mathrm{ph}}, \epsilon_{\mathrm{n}}, \epsilon_{\mathrm{p}}, \epsilon_{\mathrm{Eg}}, \epsilon_{\mathrm{Fn}}, \epsilon_{\mathrm{Fp}}\right) \mathrm{d} \epsilon_{\mathrm{n}} \mathrm{d} \epsilon_{\mathrm{p}} \mathrm{d} \epsilon_{\mathrm{Eg}} \mathrm{d} \epsilon_{\mathrm{Fn}} \epsilon_{\mathrm{Fp}}
\end{aligned}
$$

where $E_{\mathrm{ph}}$ is the photon energy, $\epsilon_{\mathrm{Fn}}$ and $\epsilon_{\mathrm{Fp}}$ are quasi-Fermi levels for electrons and holes, $\epsilon_{\mathrm{n}}$ and $\epsilon_{\mathrm{p}}$ are electron and hole energies relative to band edges, and $I_{\mathrm{ph}}\left(E_{\mathrm{ph}}\right)$ is the spectral emission intensity. For modeling of the spectra, a band-edge emission was supposed (see the discussion in the introductory part and in the previous paper, ref 11). The carrier distributions are obtained by the product of a parabolic band state density distribution $D_{\mathrm{C}, \mathrm{V}}(\epsilon) \sim \epsilon^{1 / 2}$ and Fermi occupation function $\rho_{\mathrm{C}, \mathrm{V}}\left(\epsilon_{\mathrm{n}, \mathrm{p}}, \epsilon_{\mathrm{Fn}, \mathrm{Fp}}\right)=D_{\mathrm{C}, \mathrm{V}}(\epsilon) /\left[1+\exp \left(\left(\left(\epsilon_{\mathrm{n}, \mathrm{p}}-\epsilon_{\mathrm{Fn}, \mathrm{Fp}}\right) /\right.\right.\right.$ $k T)$ ]. To simplify the integration we can suppose for the minority carrier density distribution a delta-like function at energies close to the valence band edge. So, the integration is reduced to

$$
I_{\mathrm{ph}}\left(E_{\mathrm{ph}}\right) \sim \int \rho_{\mathrm{C}}\left(\epsilon, \epsilon_{\mathrm{Fn}}\right) P\left(E_{\mathrm{ph}}, \epsilon, \epsilon_{\mathrm{Eg}}, \epsilon_{\mathrm{Fn}}\right) \mathrm{d} \epsilon \mathrm{d} \epsilon_{\mathrm{Eg}} \mathrm{d} \epsilon_{\mathrm{Fn}}
$$

The broadening of PL peaks is caused by different phenomena: (i) local potential fluctuations, which cause fluctuations in the band edges with $P\left(E_{\mathrm{ph}}, \epsilon, \epsilon_{\mathrm{Eg}}, \epsilon_{\mathrm{Fn}}\right) \sim \delta\left(E_{\mathrm{ph}}-\epsilon_{\mathrm{Eg}}-\epsilon\right)$ $\delta\left(\epsilon_{\mathrm{Fn}}+\epsilon_{\mathrm{Eg}}-F_{\mathrm{n}}\right) \exp \left(-\left(\epsilon_{\mathrm{Eg}}-E_{\mathrm{g}}\right)^{2} / w_{\mathrm{g}}{ }^{2}\right)$; (ii) fluctuations of the Fermi level along the wire, relative to the conduction band edge with $P\left(E_{\mathrm{ph}}, \epsilon, \epsilon_{\mathrm{Eg}}, \epsilon_{\mathrm{Fn}}\right) \sim \delta\left(E_{\mathrm{ph}}-\epsilon_{\mathrm{Eg}}-\epsilon\right)$ $\delta\left(\epsilon_{\mathrm{Eg}}-E_{\mathrm{g}}\right) \exp \left(-\left(\epsilon_{\mathrm{Fn}}-F_{\mathrm{n}}\right)^{2} / w_{\mathrm{F}}^{2}\right)$; (iii) Landsberg broadening $^{33}$ due to the finite time of the final state of the electronhole recombination with $P\left(E_{\mathrm{ph}}, \epsilon, \epsilon_{\mathrm{Eg}}, \epsilon_{\mathrm{Fn}}\right) \sim \delta\left(\delta\left(\epsilon_{\mathrm{Fn}}-F_{\mathrm{n}}\right)\right.$ $\delta\left(\epsilon_{\mathrm{Eg}}-E_{\mathrm{g}}\right) \Gamma(\epsilon) /\left(\Gamma(\epsilon)^{2}+\left(\epsilon-E_{\mathrm{ph}}+E_{\mathrm{g}}\right)^{2}\right)$. Here $\delta(\epsilon)$ is the delta function, while $F_{\mathrm{n}}$ and $E_{\mathrm{g}}$ are mean values of the Fermi level for electrons and the band gap energy, respectively. Landsberg pointed out that after recombination, a hole in the Fermi sea continues to exist for a very short time and therefore there is an uncertainty in the energy of the final state of the recombination process. The result is an uncertainty of the emitted photon energy, too. For fluctuations of band edge or Fermi level position, a Gauss distribution was used. The Landsberg broadening is described by a Lorentz function with a width $\Gamma$ depending on energy.$^{33}$ Of course, in the general case a superposition of these phenomena should be taken into account. In Figure 5a, fit results are illustrated for three different broadening functions for a PL spectrum at $20 \mathrm{~K}$ for sample FSNW31. All considered broadening types describe quite well the long energy tail of the spectrum. Our main interest in modeling the spectral region close to the Fermi level aims at a shape analysis for finding the position of the Fermi level and the related electron concentration. For sample characterization, we chose the simplest model: that of Fermi level fluctuations. This model cannot describe the low energy tail, but it is more adequate for the broadening effect close to the Fermi level. From the fit, the mean value of the energy gap $E_{\mathrm{g}}$ is also estimated. However, due to fluctuations which are present in wires and included in modeling, relatively large errors in estimation of model parameters can be expected. These values are rather scattered as can be seen in Figure 5c, for example.

The Fermi energy relative to the band edge gives the electron concentration. In Figure 5b, a correlation of the PL integral intensity with the calculated electron concentration is shown. The measurements are performed at $20 \mathrm{~K}$ with a laser power of $50 \mathrm{~mW}$. The inset illustrates the difference between spectra normalized for comparison of the low energy tail, for two extreme cases of wires FSNW31 and FSNW33 

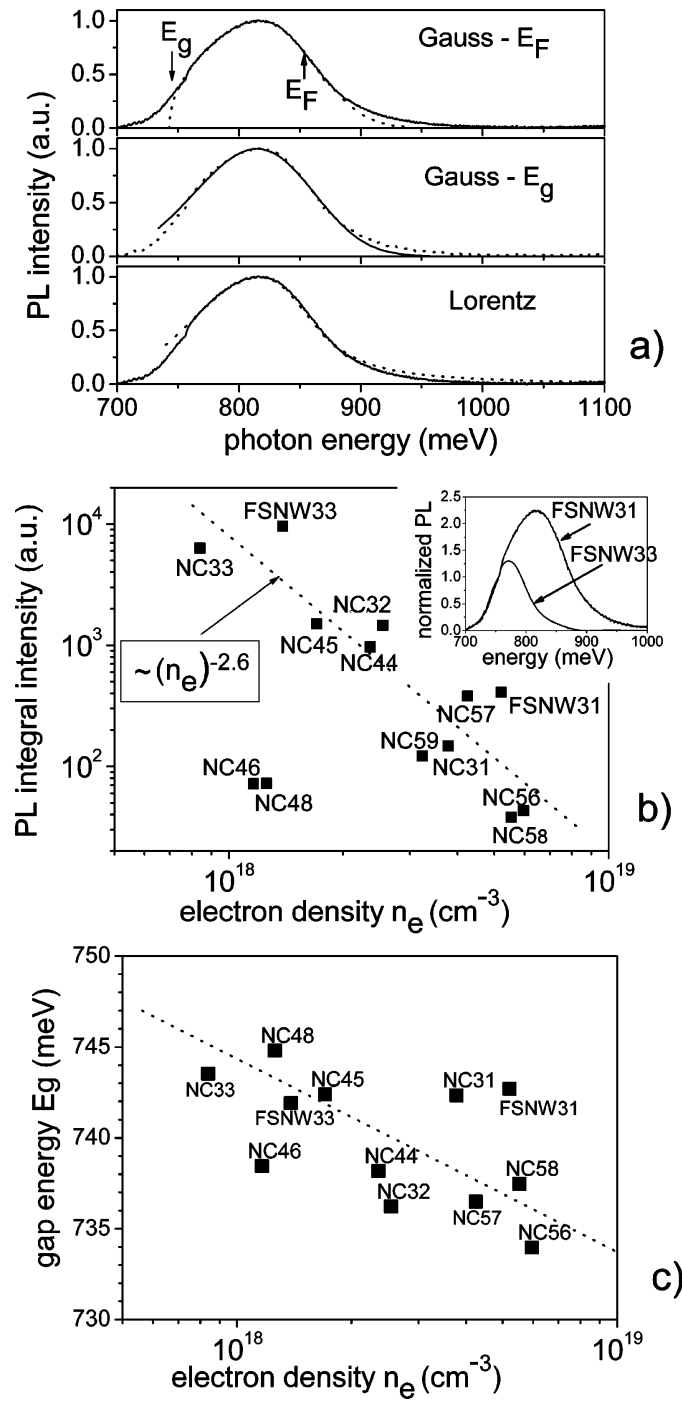

Figure 5. (a) Examples of fit results using different broadening phenomena (continuous lines are for experimental curves, dotted lines are for computed curves). (b and c) Correlation of the computed electron concentration $n_{\mathrm{e}}$ with the PL integral intensity and band gap energy $E_{\mathrm{g}}$, respectively. The inset in part b shows an example of two normalized PL spectra of samples with different electron concentrations (samples FSNW31 and FSNW33).

with high and low carrier concentration, respectively. The electron concentration was computed for a parabolic band with an electron effective mass of $0.1 \mathrm{~m}_{0}{ }^{23} \mathrm{We}$ can see that the PL integral intensity decreases with carrier concentration as a power function $\sim n^{-2.6}$. This is a stronger dependence than for a dominant Auger recombination, for which a dependence of $n^{-1}$ is expected. We are forced to conclude that the PL efficiency decreases not only due to the increase of the electron concentration but also because of other nonradiative recombination processes, as for example recombination at the wire surface. The calculated correlation in Figure $5 \mathrm{~b}$ might also be influenced by errors in the concentration evaluation due to the nonparabolicity of the conduction band. ${ }^{23}$

The dependence of the band gap energy on carrier concentration shows a tendency of a normal narrowing with electron density (Figure 5c). The band gap values are obtained in a narrow range $732-746 \mathrm{meV}$, in good agreement with the value reported recently in the literature. ${ }^{25}$

The estimated values of band gap energy and electron concentration are also given in Table 1 for samples obtained with different growth parameters. One can see in this table that, with In flux, the energy gap increases slightly, while the electron concentration decreases, for the same deposition temperature and time of $475{ }^{\circ} \mathrm{C}$ and $2 \mathrm{~h}$, respectively. The smaller electron concentration of $8 \times 10^{17} \mathrm{~cm}^{-3}$ was found for higher deposition temperature (sample NC33 deposited at $525{ }^{\circ} \mathrm{C}$ ). Higher values of $\sim 6 \times 10^{18} \mathrm{~cm}^{-3}$ were found for shorter deposition times and low In fluxes.

PL spectra of FSNWs and of as grown samples are in general different because light emissions of the substrate and a substrate influence on InN emission are avoided in FSNWs. More specific this means intrinsic and defect-induced emissions in the Si substrate or emissions from the free surface of the substrate exposed to the deposition atmosphere, radiative recombinations at $\mathrm{InN} / \mathrm{Si}$ interface and a substrate influence on InN nanocolumn emission due to strain, which affects the band gap at the column bottom. These spectra can also be different because of a random orientation of wires in FSNW samples. As a consequence bottom parts of the columns are illuminated with equal probability as top parts. If we compare the fit results for the FSNW samples and their corresponding as-deposited samples, we can see that the significant differences concern the electron density. This is attributed to an increased contribution of FSNWs in the PL spectra of the bottom part of the columns, which has higher doping. We have shown in ref 11 , that the PL efficiency increases with the column length, because of a higher quality of the upper part of the columns. A higher "intrinsic" doping due to misfit induced defects and $\mathrm{Si}$ segregation can be expected at the bottom part of the columns, similar to the case of $\mathrm{GaN}$ grown on $\mathrm{Si}(111))^{34,35}$ A larger number of structural defects observed in TEM images can explain this result. The relative influence of the strain effect on the band gap within the base region seems to be less important for the observed blue shift in PL spectra of FSNWs compared to as-deposited samples.

To conclude, the shape analysis of the PL spectra gives valuable information about the intrinsic properties of $\mathrm{InN}$ nanowires, the band gap, and the electron concentration. For different growth conditions, major variations of $\mathrm{InN}$ nanowire properties are due to changes in the equilibrium carrier concentration. The optical band gap and the electron concentration of unintentionally doped samples were found in the range $730-750 \mathrm{meV}$ and $8 \times 10^{17}$ to $6 \times 10^{18} \mathrm{~cm}^{-3}$, respectively. Differences between samples are mainly due to variations of the electron concentration (intrinsic doping). A decrease of the doping level with In flux and growth temperature was observed. An increase of the growth time also reduces the intrinsic doping. The results on free-standing nanowires are in agreement with this explanation. This is understood in terms of a higher doped region at the bottom of the columns than at the top. HRTEM shows that despite a good crystallinity and $c$-axis orientation parallel to the wires 
growth direction, some point defects are observed within the bottom part of the columns.

Acknowledgment. The authors thank K. H. Deussen and H.-P. Bochem for technical support and R. Carius for valuable discussions.

\section{References}

(1) Law, M.; Goldberger, J.; Yang, P. Annu. Rev. Mater. Res. 2004, 34, 83.

(2) Mahboob, I.; Veal, T. D.; Piper, L. F. J.; McConville, C. F.; Lu, Hai; Schaff, W. J.; Furthmueller, J.; Bechstedt, F. Phys. Rev. B 2004, 69, 201307(R)

(3) Bhuiyan, A. G.; Hashimoto, A.; Yamamoto, A. J. Appl. Phys. 2003, 94, 2779.

(4) Liang, C. H.; Chen, L. C.; Hwang, J. S.; Chen, K. H.; Hung, Y. T.; Chen, Y. F. Appl. Phys. Lett. 2002, 81, 22.

(5) Tang, T.; Han, S.; Jin, W.; Liu, X.; Li, C.; Zhang, D.; Zhou, C.; Chen, B.; Han, J.; Meyyapan, M. J. Mater. Res. 2004, 19, 423

(6) Johnson, M. C.; Lee, C. J.; Bourret-Courchesnea, E. D.; Konsek, S. L.; Aloni, S.; Han, W. Q.; Zettl, A. Appl. Phys. Lett. 2004, 85, 5670.

(7) Zhang, J.; Zhang, L.; Penga, X.; Wang, X. J. Mater. Chem. 2002, 12,802 .

(8) Zhang, J.; Xu, B.; Jiang, F.; Yang, Y.; Li, J. Phys. Lett. A 2005, $337,121$.

(9) Dimakis, E.; Konstantinidis, G.; Tsagaraki, K.; Adikimenakis, A.; Iliopoulos, E.; Georgakilas, A. Superlattices Microstruct. 2004, 36, 497.

(10) Grandal, J.; Sanchez-García, M. A.; Calle, F.; Calleja, E. Phys. Status Solidi C 2005, 2, 2289.

(11) Stoica, T.; Meijers, R.; Calarco, R.; Richter, T.; Lüth, H. J. Cryst. Growth 2006, 290, 241.

(12) Lu, H.; Schaff, W. J.; Hwang, J.; Wu, H.; Yeo, W.; Pharkya, A.; Eastman, L. F. Appl. Phys. Lett. 2000, 77, 2548.

(13) Inushima, T.; Mamutin, V. V.; Vekshin, V. A.; Ivanov, S. V.; Sakon, T.; Motokawa, M.; Ohoya, S. J. Cryst. Growth 2001, 227-228, 481.

(14) Davydov, V. Yu.; Klochikhin, A. A.; Seisyan, R. P.; Emtsev, V. V.; Ivanov, S. V.; Bechstedt, F.; Furthmüller, J.; Harima, H.; Mudryi, A. V.; Aderhold, J.; Semchinova, O.; Graul, J. Phys. Status Solidi B 2002, 229, R1

(15) Tansley, T. L.; Foley, C. P. J. Appl. Phys. 1986, 59, 3241.

(16) Davydov, V. Yu.; Klochikhin, A. A. Semiconductors 2004, 38, 897.

(17) Bechstedt, F.; Furthmüller, J.; Ferhat, M.; Teles, L. K.; Scolfaro, L. M. R.; Leite, J. R.; Davydov, V. Yu.; Ambacher, O.; Goldhahn, R. Phys. Status Solidi A 2003, 195, 628.
(18) Shubina, T. V.; Ivanov, S. V.; Jmerik, V. N.; Solnyshkov, D. D.; Vekshin, V. A.; Kopev, P. S.; Vasson, A.; Leymarie, J.; Kavokin, A.; Amano, H.; Shimono, K.; Kasic, A.; Monemar, B. Phys. Rev. Lett. 2004, 92, 117407.

(19) Specht, P.; Ho, J. C.; Xu, X.; Armitage, R.; Weber, E. R.; Erni, R.; Kisielowski, C. Solid State Commun. 2005, 135, 340

(20) Miyajima, T.; Kudo, Y.; Wakahara, A.; Yamaguchi, T.; Araki, T.; Naoi, H.; Nanishi, Y. 6th International Conf. on Nitride Semiconductors, Th-SP3-6, Bremen-Germany 2005.

(21) Saito, Y.; Harima, H.; Kurimoto, E.; Yamaguchi, T.; Teraguchi, N.; Suzuki, A.; Araki, T.; Nanish, Y. Phys. Status Solidi B 2002, 234, 796.

(22) Matsuoka, T.; Okamoto, H.; Nakao, M.; Harima, H.; Kurimoto, E. Appl. Phys. Lett. 2002, 81, 1246.

(23) Wu, J.; Walukiewicz, W.; Shan, W.; Yu, K. M.; Ager, J. W., III Haller, E. E.; Lu, H.; Schaff, W. J. Phys. Rev. B 2002, 66, 201403.

(24) Nanishi, Y.; Saito, Y.; Yamaguchi, T.; Hori, M.; Matsuda, F.; Araki, T.; Suzuki, A.; Miyajima, T. Phys. Status Solidi A 2003, 200, 202.

(25) Yu, K. M.; Liliental-Weber, Z.; Walukiewicz, W.; Shan, W.; Ager, J. W., III; Li, S. X.; Jones, R. E.; Haller, E. E.; Lu, H.; Schaff, W. J. Appl. Phys. Lett. 2005, 86, 071910.

(26) Klochikhin, A. A.; Davydov, V. Yu.; Emtsev, V. V.; Sakharov, A V.; Kapitonov, V. A.; Andreev, B. A.; Lu, H.; Schaff, W. J. Phys. Status Solidi B 2005, 242, R33.

(27) Sugita, K.; Takatsuka, H.; Hashimoto, A.; Yamamoto, A. Phys. Status Solidi B 2003, 240, 421 .

(28) Yodo, T.; Yona, H.; Ando, H.; Nosei, D.; Harada, Y. Appl. Phys. Lett. 2002, 80, 968.

(29) Yin, L. W.; Bando, Y.; Goldberg, D.; Li, M. S. Adv. Mater. 2004, $16,1833$.

(30) Wu, J.; Walukiewicz, W.; Yu, K. M.; Ager, J. W. Haller, E. E.; Lu, H.; Schaff, W. J.; Saito, Y.; Nanishi, Y. Appl. Phys. Lett. 2002, 80, 3967

(31) Walukiewicz, W.; Yu, K. M.; Li, S. X.; Denlinger, J. D.; Hsu, L.; Jones, R. E.; Ager, J. W., III; Haller, E. E.; Lu, H.; Schaff, W. J. 6th International Conf. on Nitride Semiconductors, Mo-OP1-1, BremenGermany 2005

(32) Calarco, R.; Marso, M.; Richter, Th.; Aykanat, A.; Meijers, R.; Hart, A. v. d.; Stoica, T.; Lüth, H. Nano Lett. 2005, 5, 981.

(33) Landsberg, P. T. Phys. Status Solidi 1966, 15, 623

(34) Calleja, E.; Sanchez-Garcia, M. A.; Basak, D.; Sanchez, F. J.; Calle, F.; Youinou, P.; Munoz, E.; Serrano, J. J.; Blanco, J. M.; Villar, C.; Laine, T.; Oila, J.; Saarinen, K.; Hautojarvi, P.; Molloy, C. H.; Somerford, D. J.; Harrison, I. Phys. Rev. B 1998, 58, 1550.

(35) Cavallini, A.; Polenta, L.; Rossi, M.; Richter, T.; Marso, M.; Meijers, R.; Calarco, R.; Lüth, H. Defect distribution along single GaN nanowhiskers. Nano Lett. 2006, 6, DOI 10.1021/n1060332n.

NL060547X 